\title{
Hydrodynamic modulation of pluripotent stem cells
}

\author{
Krista M Fridley', Melissa A Kinney and Todd C McDevitt*1,2
}

\begin{abstract}
Controlled expansion and differentiation of pluripotent stem cells (PSCs) using reproducible, high-throughput methods could accelerate stem cell research for clinical therapies. Hydrodynamic culture systems for PSCs are increasingly being used for high-throughput studies and scale-up purposes; however, hydrodynamic cultures expose PSCs to complex physical and chemical environments that include spatially and temporally modulated fluid shear stresses and heterogeneous mass transport. Furthermore, the effects of fluid flow on PSCs cannot easily be attributed to any single environmental parameter since the cellular processes regulating self-renewal and differentiation are interconnected and the complex physical and chemical parameters associated with fluid flow are thus difficult to independently isolate. Regardless of the challenges posed by characterizing fluid dynamic properties, hydrodynamic culture systems offer several advantages over traditional static culture, including increased mass transfer and reduced cell handling. This article discusses the challenges and opportunities of hydrodynamic culture environments for the expansion and differentiation of PSCs in microfluidic systems and larger-volume suspension bioreactors. Ultimately, an improved understanding of the effects of hydrodynamics on the self-renewal and differentiation of PSCs could yield improved bioprocessing technologies to attain scalable PSC culture strategies that will probably be requisite for the development of therapeutic and diagnostic applications.
\end{abstract}

\section{Introduction}

Pluripotent stem cells (PSCs), including embryonic stem cells (ESCs) and induced pluripotent stem cells (iPSCs), are potentially unlimited cell sources for cellular therapies due to the unique capacities of PSCs to self-renew

*Correspondence: todd.mcdevitt@bme.gatech.edu

'The Wallace H. Coulter Department of Biomedical Engineering, Georgia Institute

of Technology and Emory University, 313 Ferst Drive, Atlanta, GA 30332, USA

Full list of author information is available at the end of the article indefinitely and differentiate into cells from all three germ lineages (ectoderm, mesoderm, and endoderm) [1]. Differentiation of PSCs in vitro can be induced by a variety of methods, the most common of which are in an adherent monolayer format $[2,3]$ or via formation of three-dimensional cell spheroids in suspension culture referred to as embryoid bodies (EBs) [4]. As an alternative to traditional static adherent cell culture practices that suffer from limited scalability due to surface area dependence, PSCs can be scalably expanded and differentiated in suspension cultures [2-4].

Culture systems that employ liquid motion to modulate mass transfer and shear stress, commonly referred to as hydrodynamics, include scaled-down microfluidic systems and scaled-up bioreactor cultures. Microfluidic systems are geometrically defined culture platforms that enable high-throughput screening of culture parameters, including modulation of fluid flow rates, mass transfer, and shear stress. At the other end of the spectrum, bioreactors provide a potential scalable alternative to static cultures due to increased culture volumes and the ability to readily incorporate multiple sensors for bioprocess engineering strategies that facilitate continuous monitoring and feedback control. However, hydrodynamic cultures expose PSCs to physical and chemical factors not present in static culture, such as fluid shear stress and mass transfer via convection. The influence of hydrodynamics on the self-renewal and differentiation of PSCs has therefore been examined in both microfluidic and bioreactor systems. This review describes the current status and recent advances in understanding hydrodynamic modulation of PSCs.

\section{Hydrodynamics}

Hydrodynamics is the study of physical properties of a fluid in motion, including velocity, pressure, density, and viscosity, as functions of space and time [5]. Mathematical solutions utilizing the conservation of mass, momentum, and energy can be obtained for theoretical models with respect to fluid properties and system geometries. Such solutions are readily obtainable for two-dimensional adherent cell cultures, due to defined geometries with low flow rates, which enable precise characterization of fluid flow in microfluidic systems. Owing to difficulties associated with the transfer of momentum between the 


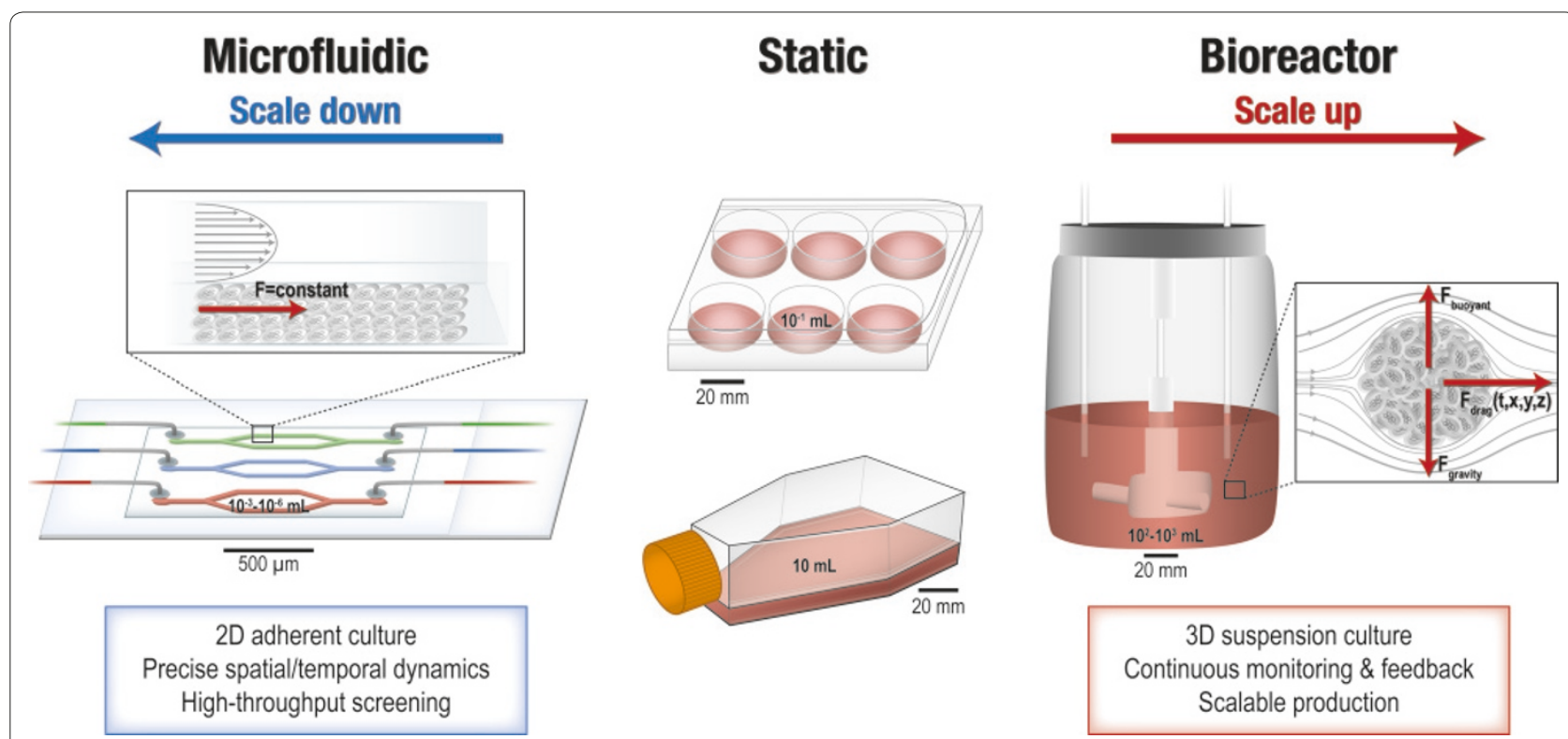

Figure 1. Comparison of hydrodynamic culture systems for pluripotent stem cell culture. Microfluidic devices provide a scale-down approach to examining hydrodynamic effects on pluripotent stem cells with precise spatial and temporal control and high-throughput formats. At the other end of the spectrum, bioreactors can be scaled up utilizing hydrodynamic systems with more complex and heterogeneous flow environments. 2D, two-dimensional; 3D, three-dimensional.

two-phase flow of solid suspension cells moving within the liquid medium, extensive work has been conducted to analyze fluid dynamics in bioreactors. Dimensionless numbers can be used to describe flow regimes; for example, the Reynolds number is used to describe laminar and turbulent flow regimes. However, important parameters, such as the mixing rate and growth factor concentrations, must be determined and similitude must be met in order to use dimensional analysis for scale-up. Experimental techniques such as particle image velocimetry have been used to characterize the three-dimensional fluid flow within bioreactors [6,7]. Computational fluid dynamics techniques can simulate fluid flow to solve equations governing fluid motion $[8,9]$, due to the difficulties associated with obtaining exact numerical solutions to the Navier-Stokes equations for turbulent flow. The complexities of hydrodynamic conditions, including intricate geometries, and spatial and temporal variations of turbulent flow, create challenges for examining the specific effects of individual hydrodynamic parameters on stem cell expansion and differentiation.

Hydrodynamic culture systems include microfluidic systems and bioreactors that employ external agitation (rotating wall or orbiting vessels) or internal agitation (stirred-flask/impeller bioreactors) (Figure 1). Although hydrodynamic effects can be broadly categorized as physical and chemical, the cellular processes regulating the balance of self-renewal and differentiation are often interconnected and thus cannot easily be attributed to a single parameter. Additionally, different culture systems exhibit complex changes in multiple parameters of the fluid flow and shear stress profiles, making it difficult to directly compare the hydrodynamic effects on stem cells between different bioreactors. For example, stirred flasks create turbulent flows (Reynolds number $>1,000$ ) with high shear stress $\left(\tau>1 \mathrm{dyn} / \mathrm{cm}^{2}\right)$ whereas rotating wall vessels maintain laminar flows (Reynolds number <100) with low shear stress $\left(\tau<1 \mathrm{dyn} / \mathrm{cm}^{2}\right)$, and exact values of Reynolds number and shear stress vary with rotation speed or location in the culture system $[6,10,11]$. Microfluidic systems with laminar flows (Reynolds number $<100)$ and physiological shear stresses $\left(\tau<20 \mathrm{dyn} / \mathrm{cm}^{2}\right)$ are ideal for high-throughput screening and mechanistic studies with precise control and manipulation of fluid properties [12-14]. In contrast, bioreactor systems are generally more amenable to scale-up in bioprocessing; however, the caveat is that hydrodynamic properties become increasingly heterogeneous as the reactor volume increases. Ultimately, microfluidic systems may provide improved understanding of important hydrodynamic culture effects on PSCs, which can then be translated to larger-volume bioreactors for scalable, bioprocessing applications.

\section{Hydrodynamics at the microscale}

Fluid flow in microfluidic systems is driven by external pressure, mechanical pumps, or pneumatic-driven pumps. Although mixing via advection between parallel 


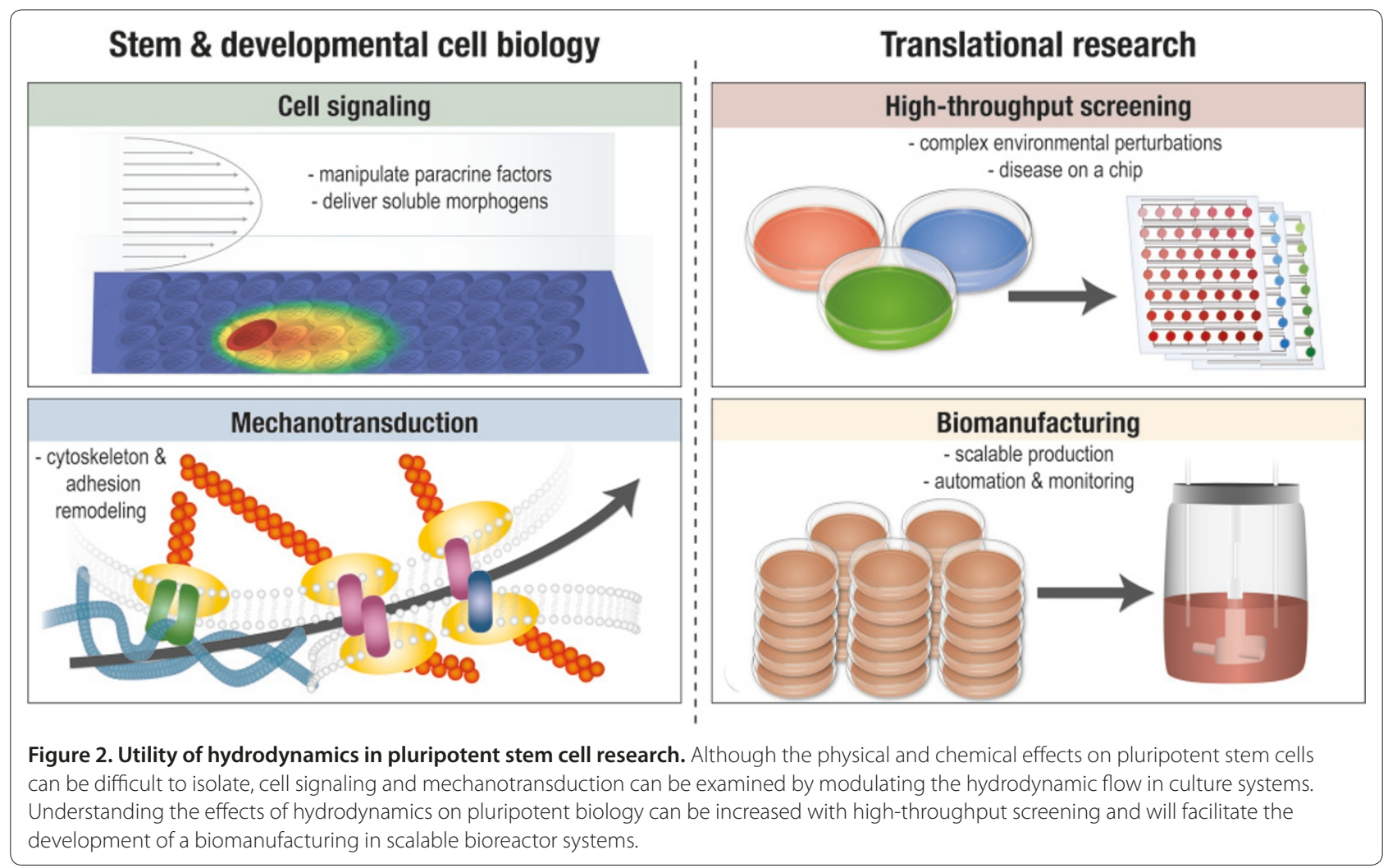

laminar flow streams is limited, microfluidic systems can generate gradients via diffusion with given morphogen(s) to replicate chemical concentration profiles comparable with those experienced by cells in vivo, thereby mimicking characteristics of embryonic development. Additionally, microfluidic systems enable more systematic characterization of heterogeneous stem cell populations via single cell analysis [15] and perturbation of cell-cell and cell-material interactions [16]. Furthermore, microfluidic devices provide high-throughput formats to examine hydrodynamic effects on PSCs in a cost-effective manner, using fewer cells and much less reagent compared with suspension bioreactors (Figure 2).

\section{Self-renewal}

In most microfluidic perfusion cultures, fresh medium flows through the system continuously, with the intent of increasing cell growth by providing nutrients and removing waste products. A microfluidic system used for expansion of human ESCs demonstrated maintenance of the pluripotency marker TG30 (CD9) over 7 days for the range of flow rates (3.6 to $44.3 \mathrm{ml} /$ hour) investigated [17]. However, only a narrow range of flow rates (20.8 and $31.3 \mathrm{ml} /$ hour) exhibited rates of human ESC expansion comparable with those for static cultures. Conditions at the lowest flow rate $(3.6 \mathrm{ml} /$ hour $)$, with a Péclet number for glucose $<1$, demonstrated a reduced cell expansion and altered morphology [17], suggesting that decreased flow rates with lower nutrient renewal and waste depletion diminish PSC expansion. In contrast, reduced expansion of cells at high flow rates (44. ml/hour) suggested that fluid shear was detrimental to cell growth.

Although mass transfer and fluid flow are often interrelated, a microfluidic platform was used to compare cell growth upon removal or delivery of cell-secreted factors $[14,18,19]$. Mouse ESC colony growth and Nanog expression were reduced when cultured under a range of shear stresses $\left(0.063\right.$ to $\left.16 \mathrm{dyn} / \mathrm{cm}^{2}\right)$ with fresh media, but not when cultured in ESC-conditioned medium at the same shear stress values, demonstrating that expansion was mediated by convective transport of soluble factors and not simply by shear stress. However, an epiblast-like phenotype, expressing Fgf5, was increased in response to shear application [14], demonstrating that shear stress elicits phenotypic changes in mouse ESCs. Even in the presence of bone morphogenetic protein 4 and leukemia inhibitory factor, which maintain the pluripotency of mouse cells in static culture, the removal of cell-secreted factors by hydrodynamic perfusion inhibited extracellular matrix remodeling and caused mouse ESCs to spontaneously differentiate [19]. Furthermore, the importance of autocrine and paracrine factors for maintaining pluripotency has been demonstrated both computationally, based on a combination of a 
stochastic three-dimensional Brownian dynamics simulation of ligand movement and a deterministic model of ligand-mediated signaling, and experimentally, where flow-dependent changes in endogenously secreted gp130activating ligands impacted heterogeneity in signaling activation of signal transducer and activator of transcription 3 [20]. Altogether, these studies demonstrate that although shear stress alone can induce phenotypic changes, fluid flow can also modulate the transport of cell-secreted factors, thereby altering PSC pluripotency (Figure 2).

\section{Differentiation}

Shear stress is generated in vivo by blood flow throughout the vasculature, as well as in the lymphatic and glomerular systems, and therefore has been investigated for differentiation of PSCs, particularly towards hematopoietic and endothelial lineages. Before the advent of microfluidic technologies, parallel-plate chamber systems were commonly used to examine the effects of controlled shear stresses on cell physiology. Applied shear stresses comparable with physiologic levels in embryonic dorsal aorta $\left(5 \mathrm{dyn} / \mathrm{cm}^{2}\right)$ and in large vessels $\left(15 \mathrm{dyn} / \mathrm{cm}^{2}\right)$ have demonstrated increased hematopoietic [21] and endothelial [22] differentiation of mouse ESCs, respectively. Additionally, the mechanisms of shear-induced PSC responses, which lead to vascular endothelial cell-specific markers and tight junction gene expression, was mediated by cell surface heparan sulfate proteoglycan [23]. In addition to dependence on shear stress magnitude, shear stress induced a time-dependent and reversible increase in the expression of an arterial endothelial cell marker (ephrinB2) [24], indicating that cellular phenotypes may be dynamically altered, thereby suggesting significant implications for matching of in vitro culture environmental conditions with in vivo transplantation sites for the translation of PSCs in cellular therapies. Perfusion cultures also induced increased albumin secretion and urea production in human ESC-derived hepatic cells compared with static cultures [25], demonstrating the importance of hydrodynamics for generating functional differentiated cells and tissues. Although these studies establish a foundation for the isolation of shear stress effects on differentiation of PSCs, the effects of shear stress on the differentiated progeny of PSCs and PSCderived tissue constructs will also be important for tissue engineering, as they will probably be present in hydrodynamic conditions created in bioreactors as well as in vivo.

Microfluidic systems can deliver proteins and signaling molecules with precise spatial and temporal control that mimics the establishment and maintenance of concentration gradients present within developing tissues [26]. For example, decreasing concentration gradients of Wnt3a demonstrated proportional decreases in $\beta$-catenin signaling in three regions of the microfluidic device perpendicular to the delivery of Wnt3a, using (A375) cells expressing a Wnt/B-catenin reporter [26]. In addition, different cytokine solutions (sonic hedgehog with fibroblast growth factor 8 or bone morphogenetic protein 4) differentiated human ESC-derived neural progenitor cells into neuronal cell body clusters and neurite bundles proportional to Sonic hedgehog concentrations in a gradient chip device [27]. Furthermore, delivery of retinoic acid using a Y-channel device design with laminar flow of different adjacent culture media compositions resulted in hemispherical neural differentiation patterns within EBs [12]. Hence, concentration gradients presented by microfluidic devices can spatially control PSC signaling and differentiation.

As mentioned above, microfluidic devices are able to isolate the physical and chemical effects of hydrodynamic culture conditions on PSCs to further interrogate the different cellular outcomes for self-renewal or directed differentiation of PSCs $[14,18,19]$. In addition, microfluidic devices provide opportunities to explore a range of hydrodynamic parameters in a systematic manner by utilizing arrays of geometric, configuration, and operating parameters, $[13,28,29]$. The ability of microfluidic systems to systematically examine the physical and chemical effects of hydrodynamic culture parameters provides a better understanding of the biological effects on PSCs for engineering of hydrodynamic microenvironments, which is difficult in the more complex and heterogeneous flow environments of bioreactor systems.

\section{Hydrodynamics in bioprocessing}

Although microfluidic systems allow increased spatial and temporal control of fluid shear and soluble factors, differentiation of PSCs in suspension does not rely on sampling small cell numbers from heterogeneous stem cell populations and is not limited by surface area, and therefore offers several advantages for scalable differentiation. Consequently, scale-up using suspension bioreactors is favorable for clinical applications in which the demand for large quantities $\left(>10^{7}\right)$ of cells are anticipated $[30,31]$. Hydrodynamic conditions imparted within bioreactors are intended to provide enhanced mass transfer and to minimize zones of shear stress, which may cause physiological perturbations or physical damage to cells.

\section{Self-renewal}

Stem cell expansion in suspension bioreactors is typically accomplished via seeding of PSCs on microcarriers $[32,33]$ or by the formation of three-dimensional multicellular aggregates [32,34]. Microcarriers provide a high surface area per volume for attachment of PSCs in suspension culture; however, the substrata provided by 
the microcarriers can influence PSC attachment, growth, and pluripotency $[32,35]$. Expansion of human ESCs as aggregates with optimized bioprocessing parameters including cell inoculation density, enzymatic dissociation medium, and rotation speed - resulted in a rapid scaleup strategy that produced clinically relevant numbers of human PSCs $\left(\sim 2 \times 10^{9}\right.$ cells $)$ over a 1-month period [36]. Additionally, monitoring and independent control of multiple vessels in parallel enabled the identification of important bioprocess parameters for PSC expansion, including cell inoculation density and aggregate formation [37]. Alternatively, antibody blocking of E-cadherinmediated cellular aggregation allowed the proliferation of mouse ESCs as single cells in shake-flask bioreactors [38]. Although expansion of PSCs using blocking antibodies may not be cost-effective, the use of small molecule inhibitors to similarly decrease cell aggregation may offer advantages by avoiding enzymatic passaging and limiting unwanted spontaneous differentiation of large multicellular aggregates, as shown by Rho-associated protein kinase inhibitor in combination with the application of heat shock to enhance cell survival and increase overall cell yield of human ESC lines [39].

Interestingly, hydrodynamic culture systems have demonstrated increased maintenance of pluripotency in comparison with static cultures during differentiation [40]. Bioreactor-derived chondrogenic, osteogenic, and cardiomyocytic cells differentiated from mouse ESCs augmented the development of teratomas upon implantation compared with those differentiated in static culture [41], illustrating the persistence of pluripotency during hydrodynamic differentiation. Although the mechanism for bioreactor maintenance of self-renewal is not clear, hydrodynamics appear to support increased PSC selfrenewal compared with static culture systems, even when using standard differentiation protocols. Hydrodynamicmediated self-renewal may offer advantages for the expansion of PSCs but also highlights the potential safety concerns regarding the potential tumorigenecity of differentiated PSC populations upon transplantation.

As described previously, perfusion provides continuous renewal of nutrients and elimination of waste products as well as introducing an additional external fluid flow term within bioreactors that can further modulate the fluid shear and transport profiles. The number of human ESCs was increased by $70 \%$ in monolayer perfusion culture compared with static conditions [42], which indicates that the continuous supply of nutrients and growth factors from conditioned medium can significantly enhance PSC expansion, thereby supporting the scalability of principles described within microfluidic systems. In addition to the influence of nutrients and signaling factors, controlling dissolved oxygen in a perfused stirred tank system improved the final yield of expanded human
ESCs by 12-fold compared with traditional static culture [43], suggesting the importance of concurrent monitoring and control of the physiochemical environment for PSC culture.

\section{Differentiation}

Suspension hydrodynamic cultures have been utilized to promote PSC aggregation to form EBs and subsequent differentiation into each of the three germ layers. Rotary wall vessels increased the efficiency of EB formation by threefold compared with static culture, supported differentiation of human ESCs into primitive blood cells, and cartilage-like structures [44], as well as improving differentiation toward cardiomyocytes over static cultures [45]. Additionally, improved homogeneity of EB morphology and size have been demonstrated in stirred [46] and rotary [47] orbital cultures, which may be factors implicated in the enhanced standardization of differentiation within hydrodynamic cultures.

Shear stresses can be modulated within a particular culture system by altering the rotation speed to investigate the effects of fluid shear on PSC differentiation. Within rotary orbital shakers, changes in rotation speed varied the nominal shear stress $\left(\sim 0.7\right.$ to $\left.2.5 \mathrm{dyn} / \mathrm{cm}^{2}\right)$ and modulated the EB size, morphology, and gene expression of mouse ESCs $[47,48]$, suggesting that subtle changes in hydrodynamic properties can affect the relative proportions of differentiated cell phenotypes. Decreases in rotation speed (10 to $20 \mathrm{rpm}$ ) also decreased EB size in rotary wall vessels [49]. Additionally, changes in stirring speed in a bench-scale bioreactor demonstrated an optimal speed (65 rpm) for increased cell yields and cardiomyogenic differentiation [50]. However, changes in rotation speed in stirred-tank systems did not alter the efficiencies of osteogenic and chondrogenic [51] or hematopoietic [52] differentiation. These results suggest that modulation of hydrodynamic parameters via changes in agitation speeds within bioreactor culture systems can differentially alter PSC differentiated phenotypes.

While many studies have focused on variation of mixing parameters within a single hydrodynamic system, PSC differentiated phenotypes can also be modulated within different bioreactor configurations; a spinner flask with glass ball impeller improved human ESC differentiation towards cardiac and endothelial lineages over rotary wall, rotary orbital, and paddle-impeller spinnerflask systems [53]. In addition, differentiation to $\mathrm{ckit}^{+}$or sca $1^{+}$progenitor cell populations from mouse ESCs differed significantly between hydrodynamic environments created in spinner-flask or rotary wall vessels [52]. In perfusion bioreactors, human ESC aggregates exhibited similar characteristics to cells differentiated in vivo at the histological as well as transcriptional levels, compared with suspension EB cultures [54], highlighting 
a potential in vitro model that is comparable with in vivo multi-lineage differentiation. Although the precise mechanisms whereby hydrodynamic cultures modulate PSC cultures remain ill-defined, these studies indicate that the physical and/or chemical effect parameters introduced by hydrodynamic mixing in bioreactors modulate differentiation towards specific lineages.

The numerous factors governing PSC differentiation are often complex and interconnected; changes in the hydrodynamic environment therefore probably alter multiple biological parameters simultaneously. Isolating such parameters could offer a more mechanistic understanding of how PSC differentiation is specifically mediated by changes in the flow conditions, similar to the microfluidic studies described above. For example, it is unclear whether the previously discussed changes in PSC differentiation are due to the hydrodynamic environment or the EB size, both of which are modulated by rotation speed. Therefore, by maintaining uniform populations of size-controlled EBs in different rotation speeds (45 and $65 \mathrm{rpm}$ ) to isolate the impact of EB size on differentiation, EBs exhibited increased uniformity of differentiation, with subtle changes in the differentiation toward certain lineages [55]; however, despite the modest differences observed when normalizing for EB size and formation, the persistence of subtle phenotype changes indicates some role for hydrodynamics in modulating PSC fate decisions.

\section{Induced pluripotent stem cells and reprogramming}

Both mouse and human somatic cells have been reprogrammed to yield pluripotent cells [56-58]; however, the large-scale generation of iPSCs has been limited, at least in part due to the scalable limitations of twodimensional, static cultures and the inherent inefficiency of most reprogramming methods. In addition to the advantages mentioned above for the utility of scalable hydrodynamic ESC cultures, the ability to rapidly reprogram and expand iPSCs offers additional advantages, including providing autologous sources of PSCs and enabling novel types of in vitro models of complex genetic diseases [59]. The expansion and differentiation of iPSCs have therefore been explored in hydrodynamic cultures, similar to ESCs described above, such as orbital shakers and stirred flasks [36,60-62].

In addition, somatic cells have been reprogrammed directly in suspension culture conditions. Mouse embryonic fibroblasts were transduced using retroviral vectors expressing reprogramming factors (Oct4, Sox2, Klf4 and c-Myc); after 12 days, stirred suspension cultures generated 50 million alkaline phosphatase-positive cells in suspension compared with only 4 million cells in adherent cultures [63]. The generation of iPSCs was also increased using doxycyline inducible reprogramming in suspension cultures to encourage apoptosis of incompletely reprogrammed cells, which cannot survive in suspension [64]. Overall, these studies demonstrate that suspension cultures can facilitate reprogramming without repeated selection via passaging of adherent cells, and therefore may improve the selection of iPSCs by taking advantage of the inability of anchorage-dependent cell populations to survive in suspension culture.

\section{Integrated bioprocessing}

One potential advantage of suspension bioreactor systems is the development of integrated processes for the scalable generation of therapeutic cell populations (Figure 2). Integrating expansion and lineage-specific differentiation has been explored in several hydrodynamic culture systems [65-67]. In stirred cultures, human ESCs on microcarriers demonstrated proliferation comparable with that of human ESCs in dishes followed by efficient transition to definitive endoderm after exposure to soluble stimuli in the bulk medium [65]. In rotary wall cultures, expansion integrated with osteogenic differentiation generated cell growth and matrix formation of mouse ESCs encapsulated in alginic acid and gelatin hydrogels [66]. Integrated bioprocessing techniques exhibited utility for expansion and cryopreservation of pluripotent human ESCs, whereby the combination of cell microencapsulation with microcarrier technology improved production and storage of human ESCs with high expansion ratios (an approximately 20 -fold increase in cell concentration) and high cell recovery yields ( $>70 \%$ ) after cryopreservation [67].

Another potential use of hydrodynamic systems is for the efficient disaggregation of compact aggregates into single cells, for applications that require subsequent culture, purification, or transplantation steps. A capillary flow device capable of dissociating EBs was developed by exposing the ESC multicellular aggregates to different flow velocities (3.1, 6.2 and $8.1 \mathrm{~m} / \mathrm{second})$; however, this process resulted in the death of up to $50 \%$ of the released cells [68]. Interestingly, single cells demonstrated high viability (96\%) when exposed to the highest velocity $(8.1 \mathrm{~m} / \mathrm{second})$, indicating that the loss of viability is related to the dissociation of cellular adhesions rather than shear stress-mediated $\left(25,50\right.$ and $\left.65 \mathrm{~N} / \mathrm{m}^{2}\right)$ apoptosis. Using principles of fluid flow to dissociate single cells from cellular aggregates or microcarriers could provide a higher throughput and less cytotoxic method than enzymatic dissociation techniques. Ultimately, integrating techniques for the expansion, differentiation, and cryopreservation of PSCs could increase automation and efficiency for future bioprocessing applications.

To develop culture systems for good manufacturing practice (GMP) bioprocessing, the use of serum-free medium and automated, controlled systems via 
hydrodynamic bioreactors could improve the clinical translation of PSCs. The inclusion of serum in culture media creates challenges for PSC expansion and differentiation by introducing lot-to-lot variability and xenogenic antigens into the cultures [69]. However, serum can buffer mammalian cells from physical damage due to mechanical stresses created within bioreactor cultures [70,71]; stem cell culture in serum-free conditions could therefore make the cells more sensitive to hydrodynamic forces. The ability to engineer hydrodynamic culture platforms without serum was demonstrated by adjusting the medium viscosities (0.9, 40 , and 70 centipoise) using carboxymethyl cellulose; overall, more homogeneous size-controlled aggregates were generated using medium with a viscosity of 40 centipoise and an optimized rotation speed (50 rpm) [36]. The differentiation of mouse ESCs in serum-free cultures containing osteogenic cell-seeded microcarriers yielded successful incorporation into mouse (burr-hole) fractures in the tibiae without incidence of tumor formation [33,51].

A scalable, GMP platform produced 20\% myosin heavy chain and $\alpha$-actinin-expressing cardiomyocytes from human ESC [72], demonstrating efficient, scalable differentiation using GMP conditions. Cryopreserved human ESC banks created under GMP conditions in stirred cultures were optimized to increase the cell expansion rate, pluripotency, and cell yields using defined serumfree media, seeding density, and cell splitting interval [73]. Furthermore, mouse ESCs expressing Oct-4, Nanog, and SSEA-1 expanded by $85 \pm 15$-fold over 11 days in a fully controlled stirred-tank bioreactor by first optimizing the feeding regimen and cell inoculation procedure using spinner flasks [74], indicating the ability to significantly scale-up PSC expansion from laboratory-scale hydrodynamic culture systems.

The integration of defined GMP protocols within hydrodynamic cultures may provide new opportunities for PSC expansion and differentiation by removing the variability related to common laboratory culture procedures, such as the use of serum and frequency of manual cell handling.

\section{Conclusions: coupling pluripotent stem cell culture and hydrodynamics}

Dimensionless analysis and determination of the critical process parameters for each bioreactor system may direct PSC culture requirements; however, such parameters are expected to be different between bioreactors of different geometries as well as the desired cell phenotype. Additionally, the cellular processes regulating selfrenewal and differentiation cannot easily be attributed to a single parameter within hydrodynamic cultures. The behavior of hydrodynamic systems will therefore be better understood when fluid flow and cell culture characterizations can be coupled in devices to assess their interdependent influence in response to system perturbations. Decoupling hydrodynamic effects, including physical and chemical effects, from other perturbations in the microenvironment in high-throughput microfluidic systems could provide an improved understanding of the balance between the expansion and differentiation of PSCs, which can be translated to bioreactors for scalable, bioprocessing applications. Understanding the effects of hydrodynamics on pluripotent biology will enable the development of a complete bioprocess in scalable bioreactor systems for the expansion, differentiation, and subsequent storage of PSCs prior to their final intended use. Ultimately, controlled hydrodynamic processes for the high-throughput generation of cells will minimize labor-intensive multi-step approaches for applications of PSCs in cellular therapies and tissue engineering.

This article is part of a thematic series on Physical influences on stem cells edited by Gordana Vunjak-Novakovic. Other articles in the series can be found online at http://stemcellres.com/series/physical

\section{Abbreviations}

EB, embryoid body; ESC, embryonic stem cell; GMP, good manufacturing practice; iPSC, induced pluripotent stem cell; PSC, pluripotent stem cell.

\section{Competing interests}

The authors declare that they have no competing interests.

\section{Acknowledgments}

The authors are supported by funding from the National Institutes of Health (R01 GM088291 and R01 EB010061). MAK was supported by a National Science Foundation Graduate Research Fellowship and is currently supported by an American Heart Association Predoctoral Research Fellowship.

\section{Author details}

'The Wallace H. Coulter Department of Biomedical Engineering, Georgia Institute of Technology and Emory University, 313 Ferst Drive, Atlanta, GA 30332, USA. ${ }^{2}$ The Parker H. Petit Institute for Bioengineering and Bioscience, Georgia Institute of Technology, Atlanta, GA, 315 Ferst Drive, Atlanta, GA 30332, USA

\section{Published: 20 November 2012}

\section{References}

1. Martin GR: Isolation of a pluripotent cell-line from mouse embryos cultured in medium conditioned by tetratocarcinoma stem-cells. Proc Natl Acad SciU S A 1981, 78:7634-7638.

2. Ying Q-L, Stavridis M, Griffiths D, Li M, Smith A: Conversion of embryonic stem cells into neuroectodermal precursors in adherent monoculture. Nat Biotechnol 2003, 21:183-186.

3. Nakano T, Kodama H, Honjo T: Generation of lymphohematopoietic cells from embryonic stem cells in culture. Science 1994, 265:1098-1101.

4. Doetschman TC, Eistetter H, Katz M, Schmidt W, Kemler R: The in vitro development of blastocyst-derived embryonic stem cell lines: formation of visceral yolk sac, blood islands and myocardium. J Embryol Exp Morphol 1985, 87:27-45.

5. Batchelor GK: An Introduction to Fluid Dynamics. Cambridge: University Press: 1967.

6. Sucosky P, Osorio DF, Brown JB, Neitzel GP: Fluid mechanics of a spinnerflask bioreactor. Biotechnol Bioeng 2004, 85:34-46. 
7. Dusting J, Sheridan J, Hourigan K: A fluid dynamics approach to bioreactor design for cell and tissue culture. Biotechnol Bioeng 2006, 94:1196-1208.

8. Hutmacher DW, Singh H: Computational fluid dynamics for improved bioreactor design and 3D culture. Trends Biotechnol 2008, 26:166-172.

9. He JK, Li DC, Liu YX, Li X, Xu SL, Lu BH: Computational fluid dynamics for tissue engineering applications. J Mech Med Biol 2011, 11:307-323.

10. Elias CB, Desai RB, Patole MS, Joshi JB, Mashelkar RA: Turbulent shear stress - effect on mammalian cell culture and measurement using laser Doppler anemometer. Chem Eng Sci 1995, 50:2431-2440.

11. Begley CM, Kleis SJ: The fluid dynamic and shear environment in the NASA/ JSC rotating-wall perfused-vessel bioreactor. Biotechnol Bioeng 2000, 70:32-40.

12. Fung W-T, Beyzavi A, Abgrall P, Nguyen N-T, Li H-Y: Microfluidic platform for controlling the differentiation of embryoid bodies. Lab Chip 2009, 9:2591-2595.

13. Cimetta E, Figallo E, Cannizzaro C, Elvassore N, Vunjak-Novakovic G: Microbioreactor arrays for controlling cellular environments: design principles for human embryonic stem cell applications. Methods 2009, 47:81-89.

14. Toh YC, Voldman J: Fluid shear stress primes mouse embryonic stem cells for differentiation in a self-renewing environment via heparan sulfate proteoglycans transduction. Faseb J 2011, 25:1208-1217.

15. Wang DJ, Bodovitz S: Single cell analysis: the new frontier in 'omics'. Trends Biotechnol 2010, 28:281-290.

16. Prabhakarpandian B, Shen M-C, Pant K, Kiani MF: Microfluidic devices for modeling cell-cell and particle-cell interactions in the microvasculature. Microvasc Res 2011, 82:210-220.

17. Titmarsh D, Hidalgo A, Turner J, Wolvetang E, Cooper-White J: Optimization of flowrate for expansion of human embryonic stem cells in perfusion microbioreactors. Biotechnol Bioeng 2011, 108:2894-2904.

18. Blagovic K, Kim LLY, Voldman J: Microfluidic perfusion for regulating diffusible signaling in stem cells. PLoS One 2011, 6:e22892.

19. Przybyla LM, Voldman J: Attenuation of extrinsic signaling reveals the importance of matrix remodeling on maintenance of embryonic stem cell self-renewal. Proc Natl Acad Sci U S A 2012, 109:835-840,

20. Moledina F, Clarke G, Oskooei A, Onishi K, Gunther A, Zandstra PW: Predictive microfluidic control of regulatory ligand trajectories in individual pluripotent cells. Proc Natl Acad Sci U S A 2012, 109:3264-3269.

21. Adamo L, Naveiras O, Wenzel PL, McKinney-Freeman S, Mack PJ, GraciaSancho J, Suchy-Dicey A, Yoshimoto M, Lensch MW, Yoder MC, GarcíaCardeña G, Daley GQ: Biomechanical forces promote embryonic haematopoiesis. Nature 2009, 459:1131-1135.

22. Ahsan T, Nerem RM: Fluid shear stress promotes an endothelial-like phenotype during the early differentiation of embryonic stem cells. Tissue Eng Part A 2010, 16:3547-3553.

23. Nikmanesh M, Shi Z-D, Tarbell JM: Heparan sulfate proteoglycan mediates shear stress-induced endothelial gene expression in mouse embryonic stem cell-derived endothelial cells. Biotechnol Bioeng 2012, 109:583-594.

24. Masumura T, Yamamoto K, Shimizu N, Obi S, Ando J: Shear stress increases expression of the arterial endothelial marker ephrinB2 in murine ES cells via the VEGF-notch signaling pathways. Arterioscl Thromb Vasc 2009, 29:2125-2131

25. Miki T, Ring A, Gerlach J: Hepatic differentiation of human embryonic stem cells is promoted by three-dimensional dynamic perfusion culture conditions. Tissue Eng Part C Methods 2011, 17:557-568.

26. Cimetta E, Cannizzaro C, James R, Biechele T, Moon RT, Elvassore N, VunjakNovakovic G: Microfluidic device generating stable concentration gradients for long term cell culture: application to Wnt3a regulation of beta-catenin signaling. Lab Chip 2010, 10:3277-3283.

27. Park JY, Kim S-K, Woo D-H, Lee E-J, Kim J-H, Lee S-H: Differentiation of neural progenitor cells in a microfluidic chip-generated cytokine gradient. Stem Cells 2009, 27:2646-2654.

28. Figallo E, Cannizzaro C, Gerecht S, Burdick JA, Langer R, Elvassore N, VunjakNovakovic G: Micro-bioreactor array for controlling cellular microenvironments. Lab Chip 2007, 7:710-719.

29. Abaci HE, Devendra R, Soman R, Drazer G, Gerecht S: Microbioreactors to manipulate oxygen tension and shear stress in the microenvironment of vascular stem and progenitor cells. Biotechnol Appl Biochem 2012, 59:97-105.

30. Giordano A, Galderisi U, Marino IR: From the laboratory bench to the patients bedside: an update on clinical trials with mesenchymal stem cells. J Cell Physio/ 2007, 211:27-35.
31. Stiff PJ, Micallef I, Nademanee AP, Stadtmauer EA, Maziarz RT, Bolwell BJ, Bridger G, Marulkar S, Hsu FJ, DiPersio JF: Transplanted CD34(+) cell dose is associated with long-term platelet count recovery following autologous peripheral blood stem cell transplant in patients with non-Hodgkin lymphoma or multiple myeloma. Biol Blood Marrow Transplant 2011, 17:1146-1153

32. Fok EYL, Zandstra PW: Shear-controlled single-step mouse embryonic stem cell expansion and embryoid body-based differentiation. Stem Cells 2005, 23:1333-1342.

33. Alfred R, Radford J, Fan J, Boon K, Krawetz R, Rancourt D, Kallos MS: Efficient suspension bioreactor expansion of murine embryonic stem cells on microcarriers in serum-free medium. Biotechnol Progr 2011, 27:811-823.

34. Zweigerdt R, Olmer R, Singh $H$, Haverich A, Martin U: Scalable expansion of human pluripotent stem cells in suspension culture. Nat Protoc 2011, 6:689-700.

35. Chen AKL, Chen XL, Choo ABH, Reuveny S, Oh SKW: Critical microcarrier properties affecting the expansion of undifferentiated human embryonic stem cells. Stem Cell Res 2011, 7:97-111.

36. Abbasalizadeh S, Larijani MR, Samadian A, Baharvand H: Bioprocess development for mass production of size-controlled human pluripotent stem cell aggregates in stirred suspension bioreactor. Tissue Eng Part C Methods 2012. doi: 10.1089/ten.TEC.2012.0161. [Epub ahead of print]

37. Olmer R, Lange A, Selzer S, Kasper C, Haverich A, Martin U, Zweigerdt R: Suspension culture of human pluripotent stem cells in controlled, stirred bioreactors. Tissue Eng Part C Methods 2012. doi: 10.1089/ten.TEC.2011.0717. [Epub ahead of print]

38. Mohamet L, Lea ML, Ward CM: Abrogation of E-cadherin-mediated cellular aggregation allows proliferation of pluripotent mouse embryonic stem cells in shake flask bioreactors. PLoS One 2010, 5:e12921.

39. Singh H, Mok P, Balakrishnan T, Rahmat SNB, Zweigerdt R: Up-scaling single cell-inoculated suspension culture of human embryonic stem cells. Stem Cell Res 2010, 4:165-179.

40. Shafa M, Krawetz R, Zhang Y, Rattner JB, Godollei A, Duff HJ, Rancourt DE: Impact of stirred suspension bioreactor culture on the differentiation of murine embryonic stem cells into cardiomyocytes. BMC Cell Biol 2011, 12:53.

41. Taiani JT, Krawetz RJ, Zur Nieden NI, Elizabeth WuY, Kallos MS, Matyas JR, Rancourt DE: Reduced differentiation efficiency of murine embryonic stem cells in stirred suspension bioreactors. Stem Cells Dev 2010, 19:989-998.

42. Fong WJ, Tan HL, Choo A, Oh SK: Perfusion cultures of human embryonic stem cells. Bioprocess Biosyst Eng 2005, 27:381-387.

43. Serra M, Brito C, Sousa MFQ Jensen J, Tostoes R, Clemente J, Strehl R, Hyllner J, Carrondo MJT, Alves PM: Improving expansion of pluripotent human embryonic stem cells in perfused bioreactors through oxygen control. J Biotechnol 2010, 148:208-215.

44. Gerecht-Nir S, Cohen S, Itskovitz-Eldor J: Bioreactor cultivation enhances the efficiency of human embryoid body (hEB) formation and differentiation. Biotechnol Bioeng 2004, 86:493-502.

45. Wang XL, Wei GF, Yu WT, Zhao YS, Yu XJ, Ma XJ: Scalable producing embryoid bodies by rotary cell culture system and constructing engineered cardiac tissue with ES-derived cardiomyocytes in vitro. Biotechnol Progr 2006, 22:811-818.

46. Cameron CM, Hu WS, Kaufman DS: Improved development of human embryonic stem cell-derived embryoid bodies by stirred vessel cultivation. Biotechnol Bioeng 2006, 94:938-948.

47. Carpenedo RL, Sargent CY, McDevitt TC: Rotary suspension culture enhances the efficiency, yield, and homogeneity of embryoid body differentiation. Stem Cells 2007, 25:2224-2234.

48. Sargent CY, Berguig GY, Kinney MA, Hiatt LA, Carpenedo RL, Berson RE, McDevitt TC: Hydrodynamic modulation of embryonic stem cell differentiation by rotary orbital suspension culture. Biotechnol Bioeng 2010 105:611-626

49. Rungarunlert S, Klincumhom N, Bock I, Nemes C, Techakumphu M, Pirity MK, Dinnyes A: Enhanced cardiac differentiation of mouse embryonic stem cells by use of the slow-turning, lateral vessel (STLV) bioreactor. Biotechnol Lett 2011, 33:1565-1573.

50. Schroeder M, Niebruegge S, Werner A, Willbold E, Burg M, Ruediger M, Field $L$, Lehmann J, Zweigerdt R: Differentiation and lineage selection of mouse embryonic stem cells in a stirred bench scale bioreactor with automated process control. Biotechnol Bioeng 2005, 92:920-933.

51. Alfred R, Taiani JT, Krawetz RJ, Yamashita A, Rancourt DE, Kallos MS: 
Large-scale production of murine embryonic stem cell-derived osteoblasts and chondrocytes on microcarriers in serum-free media. Biomaterials 2011, 32:6006-6016.

52. Fridley KM, Fernandez I, Li M-TA, Kettlewell RB, Roy K: Unique differentiation profile of mouse embryonic stem cells in rotary and stirred tank bioreactors. Tissue Eng Part A 2010, 16:3285-3298.

53. Yirme G, Amit M, Laevsky I, Osenberg S, Itskovitz-Eldor J: Establishing a dynamic process for the formation, propagation, and differentiation of human embryoid bodies. Stem Cells Dev 2008, 17:1227-1241.

54. Stachelscheid H, Wulf-Goldenberg A, Eckert K, Jensen J, Edsbagge J, Björquist P, Rivero M, Strehl R, Jozefczuk J, Prigione A, Adjaye J, Urbaniak T, Bussmann P, Zeilinger K, Gerlach JC: Teratoma formation of human embryonic stem cells in three-dimensional perfusion culture bioreactors. J Tissue Eng Regen M 2012. doi: 10.1002/term.1467. [Epub ahead of print]

55. Kinney M, Saeed R, McDevitt T: Systematic analysis of embryonic stem cell differentiation in hydrodynamic environments with controlled embryoid body size. Integr Biol 2012, 4:641-650.

56. Yu J, Vodyanik MA, Smuga-Otto K, Antosiewicz-Bourget J, Frane JL, Tian S, Nie J, Jonsdottir GA, Ruotti V, Stewart R, Slukvin II, Thomson JA: Induced pluripotent stem cell lines derived from human somatic cells. Science 2007, 318:1917-1920.

57. Takahashi K, Yamanaka S: Induction of pluripotent stem cells from mouse embryonic and adult fibroblast cultures by defined factors. Cell 2006, 126:663-676.

58. Takahashi K, Tanabe K, Ohnuki M, Narita M, Ichisaka T, Tomoda K, Yamanaka S: Induction of pluripotent stem cells from adult human fibroblasts by defined factors. Cell 2007, 131:861-872.

59. Hochedlinger K, Plath K: Epigenetic reprogramming and induced pluripotency. Development 2009, 136:509-523.

60. Amit M, Chebath J, Margulets V, Laevsky I, Miropolsky Y, Shariki K, Peri M, Blais I, Slutsky G, Revel M, Itskovitz-Eldor J: Suspension culture of undifferentiated human embryonic and induced pluripotent stem cells. Stem Cell Rev 2010, 6:248-259.

61. Shafa M, Sjonnesen K, Yamashita A, Liu S, Michalak M, Kallos MS, Rancourt DE: Expansion and long-term maintenance of induced pluripotent stem cells in stirred suspension bioreactors. J Tissue Eng Regen Med 2011, 6:462-472.

62. Zwi-Dantsis L, Mizrahi I, Arbel G, Gepstein A, Gepstein L: Scalable production of cardiomyocytes derived from c-Myc free induced pluripotent stem cells. Tissue Eng Part A 2011, 17:1027-1037.

63. Shafa M, Day B, Yamashita A, Meng G, Liu S, Krawetz R, Rancourt DE: Derivation of iPSCs in stirred suspension bioreactors. Nat Methods 2012, 9:465-466.
64. Fluri DA, Tonge PD, Song H, Baptista RP, Shakiba N, Shukla S, Clarke G, Nagy A, Zandstra PW: Derivation, expansion and differentiation of induced pluripotent stem cells in continuous suspension cultures. Nat Methods 2012, 9:509-516.

65. Lock LT, Tzanakakis ES: Expansion and differentiation of human embryonic stem cells to endoderm progeny in a microcarrier stirred-suspension culture. Tissue Eng Part A 2009, 15:2051-2063.

66. Randle WL, Cha JM, Hwang YS, Chan KL, Kazarian SG, Polak JM, Mantalaris A: Integrated 3-dimensional expansion and osteogenic differentiation of murine embryonic stem cells. Tissue Eng 2007, 13:2957-2970.

67. Serra M, Correia C, Malpique R, Brito C, Jensen J, Bjorquist P, Carrondo MJT, Alves PM: Microencapsulation technology: a powerful tool for integrating expansion and cryopreservation of human embryonic stem cells. PLoS One 2011, 6:e23212.

68. Papantoniou I, Hoare M, Veraitch FS: The release of single cells from embryoid bodies in a capillary flow device. Chem Eng Sci 2011, 66:570-581.

69. Unger C, Skottman H, Blomberg P, Dilber MS, Hovatta O: Good manufacturing practice and clinical-grade human embryonic stem cell lines. Hum Mol Genet 2008, 17:R48-R53.

70. Michaels JD, Petersen JF, McIntire LV, Papoutsakis ET: Protection mechanisms of freely suspended animal-cells (CRL 8018) from fluid-mechanical injury viscometric and bioreactor studies using serum, pluronic F68 and polyethylene-glycol. Biotechnol Bioeng 1991, 38:169-180.

71. Michaels JD, Nowak JE, Mallik AK, Koczo K, Wasan DT, Papoutsakis ET: Interfacial properties of cell-culture media with cell-protecting additives. Biotechnol Bioeng 1995, 47:420-430.

72. Lecina M, Ting SW, Choo A, Reuveny S, Oh S: Scalable platform for human embryonic stem cell differentiation to cardiomyocytes in suspended microcarrier cultures. Tissue Eng Part C Methods 2010, 16:1609-1619.

73. Chen VC, Couture SM, Ye J, Lin Z, Hua G, Huang H-IP, Wu J, Hsu D, Carpenter MK, Couture LA: Scalable GMP compliant suspension culture system for human ES cells. Stem Cell Res 2012, 8:388-402.

74. Fernandes-Platzgummer A, Diogo MM, Baptista RP, Silva CLd, Cabral JMS Scale-up of mouse embryonic stem cell expansion in stirred bioreactors. Biotechnol Progr 2011, 27:1421-1432.

\section{doi:10.1186/scrt136}

Cite this article as: Fridley KM, et al: Hydrodynamic modulation of

pluripotent stem cells. Stem Cell Research \& Therapy 2012, 3:45. 